\title{
MODELING FREE CONVECTION FLOW OF LIQUID HYDROGEN WITHIN A CYLINDRICAL HEAT EXCHANGER COOLED TO 14 K
}

\author{
S. Q Yang, M. A. Green, and W. Lau
}

Oxford University Department of Physics, Oxford OX1 3RH, United Kingdom

\begin{abstract}
A liquid hydrogen in a absorber for muon cooling requires that up to $300 \mathrm{~W}$ be removed from 20 liters of liquid hydrogen. The wall of the container is a heat exchanger between the hydrogen and $14 \mathrm{~K}$ helium gas in channels within the wall. The warm liquid hydrogen is circulated down the cylindrical walls of the absorber by free convection. The flow of the hydrogen is studied using FEA methods for two cases and the heat transfer coefficient to the wall is calculated. The first case is when the wall is bare. The second case is when there is a duct some distance inside the cooled wall.
\end{abstract}

\section{INTRODUCTION}

Neutrino factories require that muons be accelerated to high energy before they are allowed to decay into neutrinos and electrons. The muon must be cooled in an ionization-cooling channel before they can be accelerated to high energies [1,]. This cooling must occur in a time that is substantially less than the decay time for a muon $(\sim 2.1 \square \mathrm{s})$. Ionization cooling of the muons requires a large number of liquid hydrogen absorbers that remove both the transverse and longitudinal momentum from a muons beam. RF-cavities reaccelerate the muons back to their original longitudinal momentum without adding back the transverse momentum lost in the cooling process. Liquid hydrogen has been selected as an absorber material because it has twice the $\mathrm{dE} / \mathrm{dx}$ (energy loss per unit mass per square meter) of any other material. Hydrogen has only single proton in its nucleus. Thus, the coulomb scattering, which reintroduces transverse momentum into the muons, is minimized. Hydrogen is the best material for muon ionization cooling by a factor of two.

A liquid hydrogen absorber for muon cooling requires that up to $300 \mathrm{~W}$ be removed from liquid hydrogen that has been heated by an intense muon beam. The absorber is a 350-mm long cylindrical hydrogen tank with the cylinder axis in the direction of the muon beam. The cylindrical wall of the absorber is cooled with helium gas that enters in channels on the outside of the wall at $14 \mathrm{~K}$ (just above the freezing temperature for para hydrogen). The temperature of the helium gas leaving the absorber is determined by the heat put into the liquid hydrogen and the mass flow of the helium gas. The helium gas will enter the tubes on the outside of the wall at the bottom and it will leave at the top forming a counter flow heat exchanger with the hydrogen in the absorber. It has been proposed that hydrogen heated by the muon beam rises and then it is circulated down the cylinder walls of the absorber by free convection.

Free convection hydrogen absorber cooling has been studied using conventional heat transfer methods [2, 3]. Conventional heat transfer studies suggest that the inner surface of the liquid hydrogen absorber has to be extended in order to remove anywhere near the 200 to $300 \mathrm{~W}$ of beam energy that is required. The conventional heat transfer studies were done using a duct 10 to $20 \mathrm{~mm}$ from the inner wall of the absorber to improve the heat transfer to the wall. Conventional wisdom suggests that the duct can be used to direct the flow of hydrogen down and along the cylindrical wall surface to improve the heat transfer coefficient between the hydrogen flowing down the wall and the wall.

The free-convection flow of the hydrogen was studied using finite element methods is presented in this report. The temperature drop and heat transfer coefficient at the wall was calculated for various beam powers. The free convection calculations were done using a bare cylindrical wall with no duct to direct the hydrogen flow. A duct $20 \mathrm{~mm}$ from the cylindrical wall surface was added to see if the heat transfer coefficient between the liquid hydrogen and the cylindrical wall could be improved. 


\section{DESCRIPTION OF THE MODEL}

The liquid hydrogen absorber was modeled in three dimensions. The Computational Fluid Dynamic (CFD) model [4] consists of a cylindrical body that is $300 \mathrm{~mm}$ in diameter. The body length is $235 \mathrm{~mm}$. The heat from the hydrogen is transferred out of the absorber only through the body. The area of the heat transfer surface on the inner wall of the model is $0.221-\mathrm{m}^{-2}$. At the ends of the absorber are two thin windows with a diameter of $300 \mathrm{~mm}$. The thickness of the windows at their center is $180 \mathrm{~mm}$. The length of the curved window section is $57.5 \mathrm{~mm}$. The distance between the window centers is $350 \mathrm{~mm}$. The windows are adiabatic. The muon beam is modeled as a $10-\mathrm{mm}$ diameter cylindrical heat source that goes down the axis of the absorber. The length of the cylindrical heat source is $340 \mathrm{~mm}$, in order to avoid a numerical instability at the boundary between the source and the adiabatic window.

The absorber body is modeled as a 300-mm ID aluminum cylinder with a thickness of $5 \mathrm{~mm}$ and a thermal conductivity of $235-\mathrm{W} \mathrm{m}^{-1} \mathrm{~K}^{-1}$. The length of this wall is $235-\mathrm{mm}$. The outside temperature of the cylindrical wall was set at a constant value of $14 \mathrm{~K}$. The hydrogen in the CFD model starts out at an initial temperature of $17 \mathrm{~K}$. The model is run until some sort of equilibrium is reached. The hydrogen density used for the model is $71 \mathrm{~kg} \mathrm{~m}^{-3}$. The hydrogen specific heat used in the model is $9680-\mathrm{J} \mathrm{kg}^{-1} \mathrm{~K}^{-1}$.

In the cases with the duct, the duct is $3 \mathrm{~mm}$ thick and $20 \mathrm{~mm}$ from the inner wall of the cylindrical part of the absorber. The duct length is the same as the aluminum cylindrical body length. The duct is open at the top and the bottom so that hydrogen can flow into the space between the duct and the wall. The duct is located in a region that is \pm 70 degrees on either side of the horizontal centerline of the absorber body. The duct material has the properties of glass at $300 \mathrm{~K}$; density $=2500 \mathrm{~kg} \mathrm{~m}^{-3}$, specific heat $=750 \mathrm{~J} \mathrm{~kg}^{-1} \mathrm{~K}^{-1}$ and thermal conductivity $=1.4 \mathrm{~W} \mathrm{~m}^{-1} \mathrm{~K}^{-1}$. The thermal diffusivity of the duct material is nearly the same at $20 \mathrm{~K}$ as it is at $300 \mathrm{~K}$. Figure 1 shows the CFD model for the absorber.

\section{THE RESULTS OF THE SIMULATION}

The CFD model for a beam power of $30 \mathrm{~W}$ is shown in Figure 1 below. The temperatures shown in Figure 1 are global and include the temperature of the wall as well as the temperature of the liquid hydrogen that is within the absorber. The hydrogen peak temperature where the beam strikes is about $16.8 \mathrm{~K}$. At the point where the hydrogen enters the duct, the temperature is reduced to about $16.3 \mathrm{~K}$. The hydrogen temperature at the bottom that leaves the duct is about $15.6 \mathrm{~K}$.

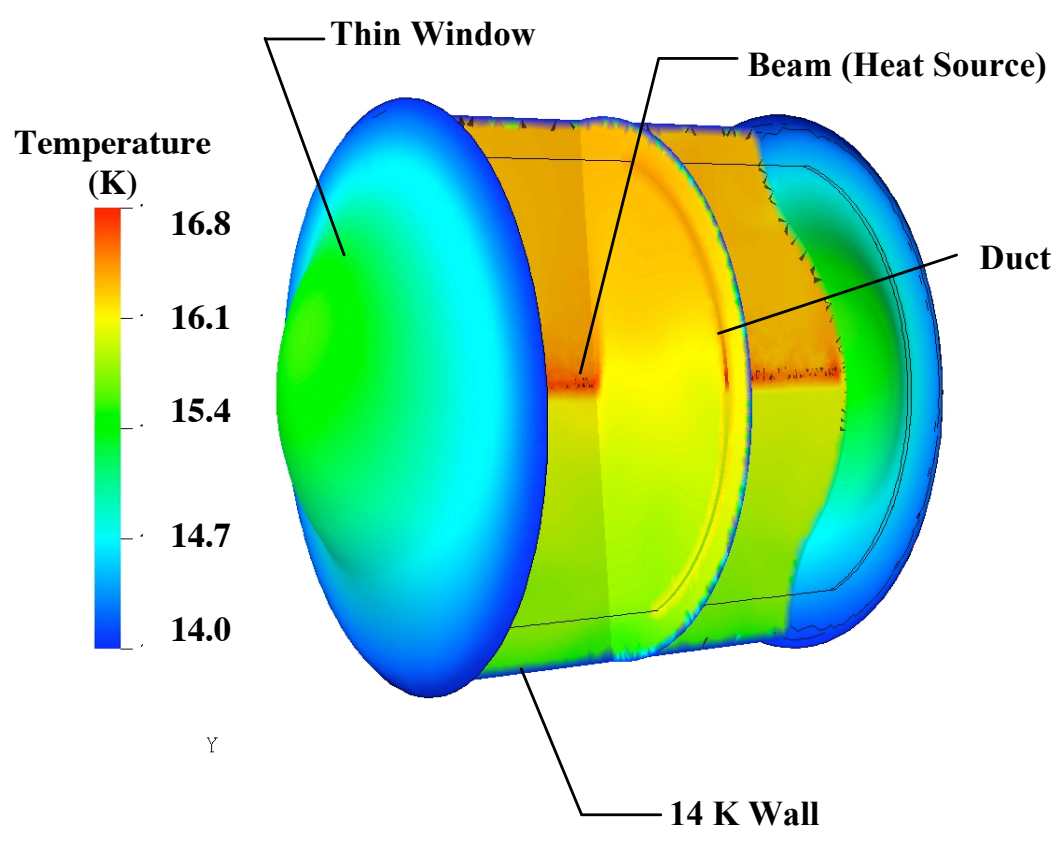

Figure 1. The CFD model of the Absorber showing the window, the duct, and heat exchanger wall. Beam power $=30 \mathrm{~W}$ 


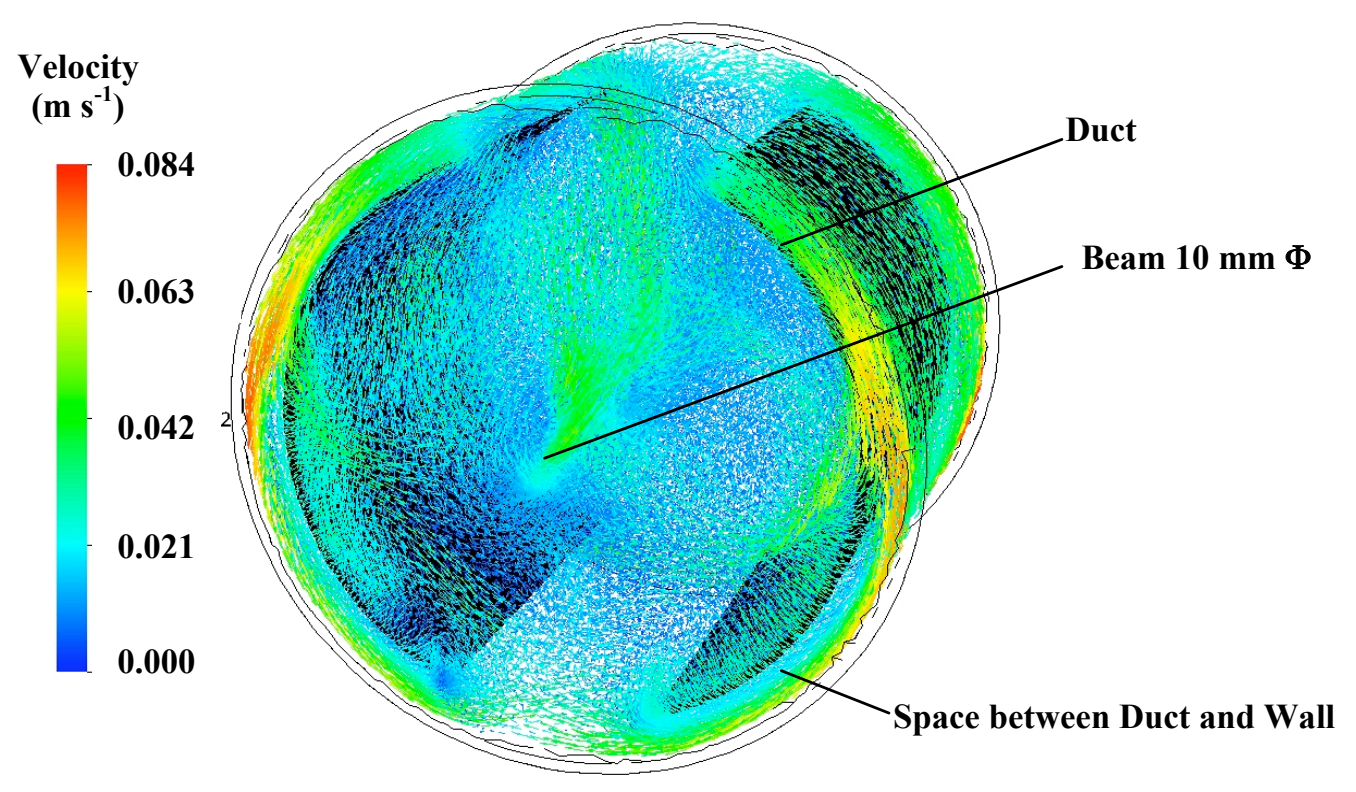

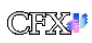

Figure 2. The velocity vector for the same case shown in Figure 1 . This case has a duct and the beam power $=30 \mathrm{~W}$

Figure 2 shows the velocity vectors for the case shown in Figure 1. One can see that the peak velocity in the system occurs within the duct, where the hydrogen flows down the wall. When there is no duct, the velocity of the flow next to the wall is between 0.01 and $0.015 \mathrm{~m} \mathrm{~s}^{-1}$. This shows that the ducting really does direct the hydrogen flow along the heat transfer surface. The down side of ducting is that the flow can be choked within the duct, because most of the driving pressure is created by the buoyancy force when the hydrogen is heated is taken up by the increased velocity between the duct and the wall.

The CFD model was run with beam powers at 30, 60, 90, 120, 150, and $200 \mathrm{~W}$. The model was run with and without a duct to direct the hydrogen flow along the wall. The model generates a temperature distribution in the liquid hydrogen and in the solid portions of the absorber (the cylindrical heat transfer surface and the windows). Velocity vectors were calculated for the liquid in the absorber. From the average hydrogen temperature along the wall one can estimate the log mean delta $\mathrm{T}$ for the heat exchanger. Using the calculated log mean delta $\mathrm{T}$, the average heat exchanger $\mathrm{U}$ factor was estimated.

For both the ducted and un-ducted absorbers, the average temperature at the top T2 and the bottom T1 of the absorber goes up with beam power (see Figure 3 left). For the duct case, T2 and T1, one can estimate the heat exchanger average $U$ factor directly. The $U$ factor calculated for the case with no duct is probably not valid for $\mathrm{Q}>120 \mathrm{~W}$. In both cases, the $\mathrm{U}$ factor goes up as $\mathrm{Q}$ increases (see Figure 3 right). The temperature rise from the bottom of the absorber to the absorber hot spot $\square \mathrm{T}$ also increases with heat load (see Figure 4 left). This temperature rise is not linear with heat load. Studies of free convection flow driven (ducted) heat exchangers suggests that $\square \mathrm{T}$ and hydrogen mass-flow both increase as $\mathrm{Q}^{0.5}[2]$. A plot of $\square \mathrm{T}$ versus $\mathrm{Q}^{0.5}$ fit to the value $\square \mathrm{T}$ at $\mathrm{Q}=90 \mathrm{~W}$ is shown in Figure 4 left for comparison.
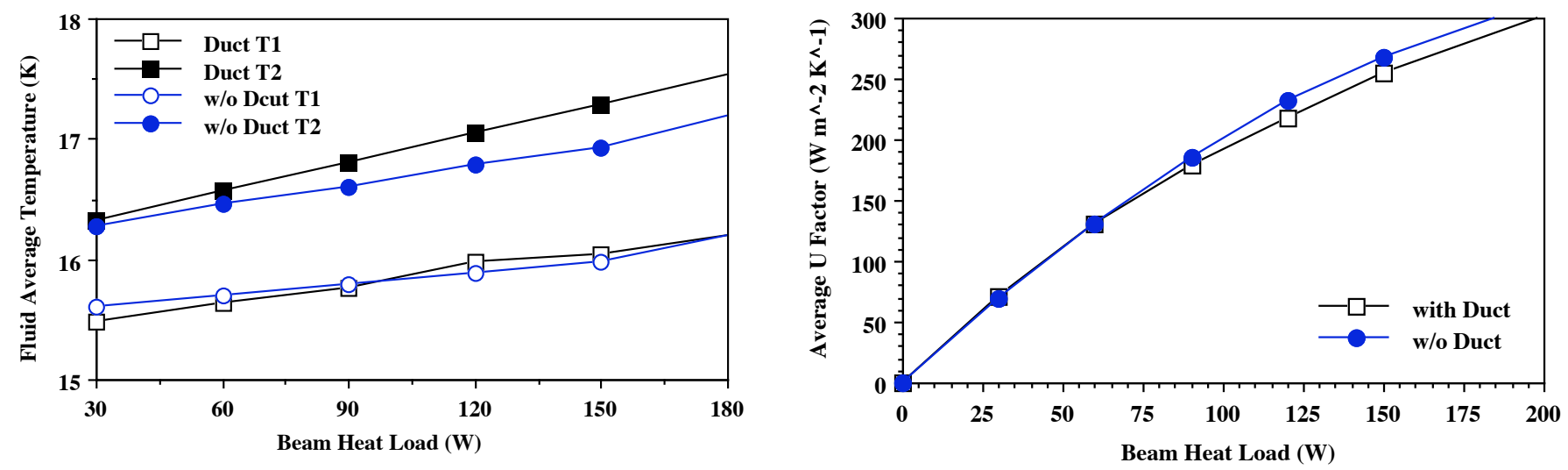

Figure 3. On the left, the fluid average temperatures at the top of the absorber T2 and bottom of the absorber T1 On the right, the average heat exchanger $\mathrm{U}$ factor versus the beam heat load Q using log-mean $\square \mathrm{T}$ calculated from $\mathrm{T} 1$ and $\mathrm{T} 2$ 

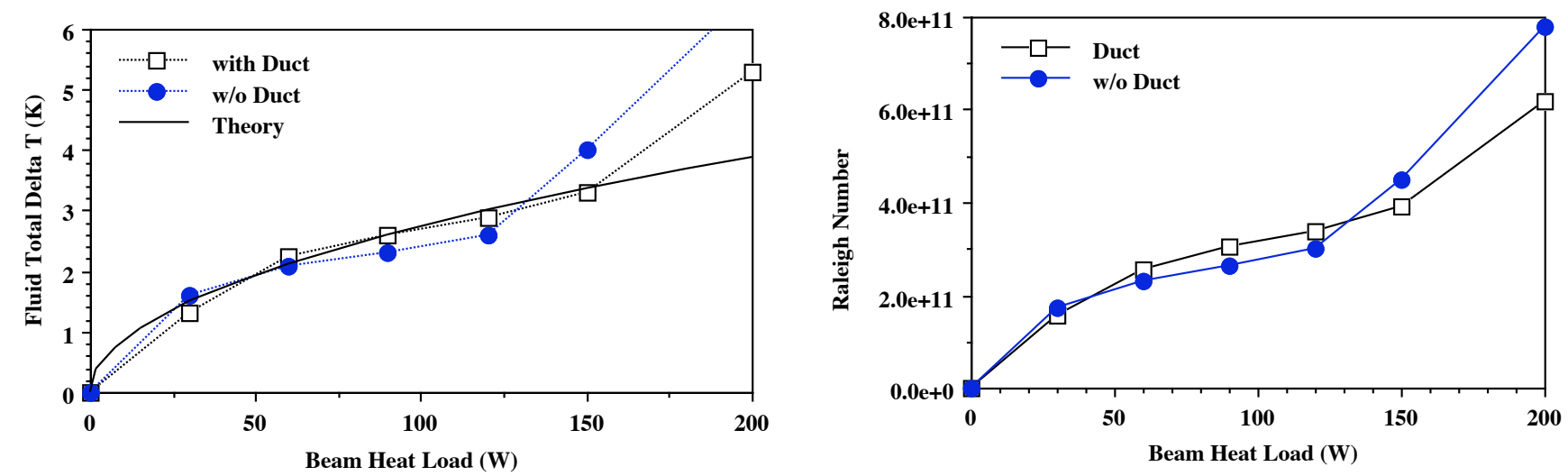

Figure 4. The total hydrogen temperature rise $\square \mathrm{T}$ and the Raleigh number of the flow as a function of the beam heat load $\mathrm{Q}$

From Figure 4 right, one can see that the calculated value of the Raleigh number has the same shape the curves for the hydrogen total $\square \mathrm{T}$ versus the beam heat load Q. At high heat loads $(>135 \mathrm{~W})$, the peak temperature of the hydrogen stream (where the beam interacts with the hydrogen) is higher for the case with no duct (see Figure 4 left). This corresponds to the $\mathrm{Q}$ where the Raleigh number is also higher (see Figure 4 right). Despite having a higher Raleigh number at $\mathrm{Q}>135 \mathrm{~W}$, the heat exchanger average heat transfer coefficient on the inner surface for the case without the duct must go down (relative to the case with the duct) for $\mathrm{Q}>135 \mathrm{~W}$. Increased mixing means that T2-T1 is lower (see Figure 3 left) for the case with no duct, but the added turbulence is not on the heat transfer surface.

\section{CONCLUDING COMENTS}

One can model a three-dimensional liquid hydrogen absorber using a CFD model. The results from the CFD model appears to be well behaved for beam heat loads Q less than $150 \mathrm{~W}$. At Q $>150 \mathrm{~W}$, the heat build up in the hydrogen will cause it to boil, even with a duct. At high heat loads, ducting does not appear to improve the calculated heat exchanger $U$ factor, but on the other hand, the duct appears to retard the temperature build up in the hydrogen due to the beam heating. The results show that the cooling performance is driven by the velocity of the flow along the wall. The ability to create a turbulent flow on the wall is the key to improving cooling. The duct appears to allow this to happen.

Further work is needed to see how the distance between the wall and the duct affect the heat transfer. The model must also be run for Gaussian beams that are up to $250 \mathrm{~mm}$ in diameter (with the same total heating in a larger beam). Finally the cooling of the wall must be properly modeled (with cold helium gas going in at the bottom at $14 \mathrm{~K}$ and warm helium gas at $17 \mathrm{~K}$ coming out of the top). It is clear that the transfer surface must be extended a factor of 3 or more, if one is going to remove $300 \mathrm{~W}$ from a helium gas cooled liquid hydrogen absorber. One good way to extend the heat transfer surface is to put part of the heat exchange surface inside the absorber. The extended heat exchanger acts as a duct on the cooled wall as well as added heat exchange surface. This study suggests that the duct can cause no harm.

\section{ACKNOWLEDGEMENTS}

This work was supported by the Oxford University Physics Department and the Particle Physics and Astronomy Research Council of the United Kingdom.

\section{REFERENCES}

1. Ozaki, S, Palmer, R. B, Zisman, M. S. and J. Gallardo, J. Eds. "Feasibility Study II of a Muon Based Neutrino Source," BNL-52623, June 2001

2, Green, M. A., "Comments on Liquid Hydrogen Absorbers for MICE,” LBNL Report LBNL-52082, Jan. 2003

3. Green, M. A., Ishimoto, S., Lau, W, and Yang, S., "A Heat Exchanger between Forced Flow Helium Gas at 14 to 18 K and Liquid Hydrogen at $20 \mathrm{~K}$ circulated by Natural Convection," Advances in Cryogenic Engineering 49, (2003)

4. CFX-4.2 Solver, AEA Technology, CFX International, Didcot OX11 0RA, United Kingdom 